\title{
Respiration-Entrained Brain Rhythms Are Global but Often Overlooked
}

\author{
Adriano B.L. Tort, ${ }^{1,3, \star}$ Jurij Brankačk, ${ }^{2,3}$ and Andreas Draguhn ${ }^{2, \star}$
}

We revisit recent evidence showing that nasal respiration entrains oscillations at the same frequency as breathing in several regions of the rodent brain. Moreover, respiration modulates the amplitude of a specific gamma sub-band $(70-120 \mathrm{~Hz})$, most prominently in frontal regions. Since rodents often breathe at delta and theta frequencies, we caution that previous studies on delta and theta power and their cross-regional synchrony, as well as on delta-gamma and theta-gamma coupling, may have detected the respiration-entrained rhythm and respiration-gamma coupling. We argue that the simultaneous tracking of respiration along with electrophysiological recordings is necessary to properly identify brain oscillations. We hypothesize that respiration-entrained oscillations aid long-range communication in the brain.

Respiration-Entrained Oscillations Are a Global Brain Rhythm

Since the pioneering work of Lord Adrian in the 1940s and 1950s, it has been well known that nasal respiration drives neuronal oscillations (see Glossary) locked to breathing cycles in the olfactory bulb (OB) $[1,2]$. In the 2000s, Fontanini and colleagues $[3,4]$ have shown that respiration-entrained oscillations propagate to the primary olfactory (piriform) cortex (Figure 1A). More recently, it has been increasingly recognized that respiration also entrains network activity in further downstream regions. For instance, oscillations in the delta frequency range $(0.5-4 \mathrm{~Hz})$ driven by respiration have been observed in local field potential (LFP) recordings from the whisker barrel cortex of awake, immobile mice [5] (Figure 1B). Respirationentrained LFP oscillations were also shown in the dorsal hippocampus of rats and mice, most prominently in the dentate gyrus (DG), during either anesthesia or awake states, with instantaneous frequency in the delta or theta $(4-12 \mathrm{~Hz})$ range depending on breathing rate [6-8] (Figure 1C). Noteworthy, prominent respiration-entrained oscillations were recently reported in the medial prefrontal cortex (mPFC) during both immobility $[9,10]$ and active behaviors [10] (Figure 1D). Other studies have extended these findings by showing that respiration-locked activity also occurs in several regions of the human brain [11,12] (Figure 1E,F).

There is now substantial evidence demonstrating that the respiration-entrained oscillations are not due to artifacts such as respiration-associated muscle activity or electrode movements. For instance, they modulate action potentials $[5-7,9,10]$ and the probability of sharp-wave ripples [13]; they are abolished following olfactory bulbectomy ([5,9], see also [13]) or when animals breathe through the trachea instead of the nose $[5,6,8]$; and in the hippocampus, they show a clear laminar profile, with maximal amplitude in DG [6-8]. Importantly, despite the overlapping frequencies (Box 1), these oscillations can be distinguished from hippocampal-generated theta by multiple features, such as the layer of maximal amplitude, coherence to respiration, dependence on nasal airflow, and insensitivity to cholinergic transmission [6-8]. Similarly, the respiration-entrained rhythm can also be distinguished from the large amplitude slow
Highlights

Nasal respiration entrains LFP oscillations in widespread regions of the rodent brain. Respiration-entrained oscillations may modulate local gamma activity.

Without tracking nasal respiration, respiration-entrained oscillations may be mistaken as delta (at low breathing rates) or theta oscillations (at high breathing rates).

Respiration and theta tend to modulate different gamma sub-bands; during REM sleep, respiration-gamma coupling decreases, while thetagamma coupling increases.

Gamma exclusively couples to respiration in the mPFC, and not to theta, which can be present at the same time.

Respiration-entrained LFP rhythms are likely to constitute a global signal aiding cross-regional communication.

${ }^{1}$ Brain Institute, Federal University of Rio Grande do Norte, Natal, RN 59056-450, Brazil ${ }^{2}$ Institute for Physiology and Pathophysiology, Heidelberg University, 69120 Heidelberg, Germany

${ }^{3}$ These authors contributed equally to this work

${ }^{*}$ Correspondence:

tort@neuro.ufrn.br (A.B.L. Tort) and andreas.draguhn@physiologie.uniheidelberg.de (A. Draguhn). 


\section{Box 1. What's in a Name?}

Brain oscillations are often named based on their frequency range. However, since respiration-entrained oscillations have highly variable peak frequency (determined by the instantaneous breathing rate), this rhythm is not well defined by the traditional nomenclature. Depending on breathing rate, the respiration-entrained rhythm may have peak frequency within the delta $(0.5-4 \mathrm{~Hz})$, theta $(4-12 \mathrm{~Hz})$, and even low beta $(12-20 \mathrm{~Hz})$ frequency ranges. Operationally, we have been defining the existence of a respiration-entrained rhythm on the basis of two requirements: (i) that the LFP power spectrum exhibits a peak at the same frequency as breathing, and (ii) that the LFP-respiration coherence spectrum also peaks at the breathing frequency. Previous work has referred to these oscillations as 'olfactory theta' [2,17], but in our opinion such a notation is not ideal because breathing rate is often outside the theta frequency. We think also that the 'olfactory theta' notation suggests a mechanistic link between respiration and theta, whereas current evidence shows both to be independent [6-8]. Moreover, it somewhat conceals the fact that olfactory regions may exhibit the 'classical' theta rhythm in addition to respiration-entrained oscillations [7,10]. In nonanesthetized animals, we reserve the term 'theta rhythm' to the well-known $6-10-\mathrm{Hz}$ activity generated in septohippocampal networks, which typically appears during locomotion and REM sleep, phase reverses across hippocampal layers, and has maximal amplitude near the hippocampal fissure $[7,25,64,68]$. In anesthetized animals, we call 'theta' the well-described oscillation of similar anatomical characteristics, though at a slower frequency $(3.5-7 \mathrm{~Hz})[6,8,73]$. For regions other than the hippocampus, we consider the LFP to exhibit theta when it has oscillations that are of the same frequency as the hippocampal theta activity, and coherent with it. Though we highlight the possibility of two distinct rhythms within the theta frequency range (i.e., the respiration-driven and the 'classical' theta), we do not exclude the possibility of yet other types of thetafrequency activity not discussed here.

oscillations in the delta range associated with thalamocortical activity and up-and-down state transitions during sleep and deep anesthesia $[8,14,15]$.

\section{Respiration-Coupled Activity as a Potential Confound in Delta and Theta Studies}

Figure 2A shows examples of respiration-entrained LFP oscillations during naturally occurring periods of awake immobility and exploration simultaneously recorded from the OB, mPFC, and parietal cortex of a mouse. Note that in immobility respiration-locked oscillations occur at delta frequency and can be readily inferred in the LFP traces from all recorded regions (respiration frequency $=\sim 3.8 \mathrm{~Hz}$ ). However, during exploration they are much harder to detect simply by visual inspection, especially in the neocortical LFPs, due to the faster respiration rate and concomitant presence of theta (theta frequency $=\sim 9.2 \mathrm{~Hz}$; respiration frequency $=\sim 11.5 \mathrm{~Hz}$ ). In this case, the two oscillations can only be confidently distinguished by analyzing the LFP power spectrum, which reveals two peaks at nearby frequencies, and by analyzing LFP coherence to respiration, which shows that one of the peaks is phase locked to breathing cycles.

Figure 2B shows an example power spectra of nasal respiration (top) and mPFC LFP signals (bottom) obtained during three periods differing in breathing rate, along with coherence spectra between the LFP and nasal respiration. The exploration example in Figure 2A is similar to the rightmost case in Figure 2B. Notice that theta oscillations can be seen in the power spectra [the leftmost case is a rapid eye movement (REM) sleep episode], but, as opposed to the respiration-entrained rhythm, theta is not coherent with nasal respiration. Moreover, while the frequency of the respiration-entrained LFP rhythm varies with breathing rate, the peak frequency of the theta rhythm is much steadier (see also [7]). Importantly, breathing rate in rodents may vary from $\sim 1 \mathrm{~Hz}$ during rest and sleep to as high as $\sim 14 \mathrm{~Hz}$ during sniffing $[16,17]$. Therefore, the frequency of the respiration-locked rhythm can be slower, similar, or faster than theta frequency (Figure 2B). This simple observation has important implications: we argue that previous work on theta-frequency oscillations may have actually detected LFP activity phase locked to respiration (e.g., see [18-24]), and not the classical theta rhythm as defined in septohippocampal circuits [25]. Even though the peak frequency of CA1 theta usually lies between 6 and $9 \mathrm{~Hz}$ in freely moving animals, most researchers define theta as oscillations between 4 and $12 \mathrm{~Hz}$, which is likely to increase the chance of 'contamination' by the respiration-entrained rhythm. Of note, the seminal work by Macrides et al. [26] and Kay

\section{Glossary}

Coherence: a measure of the stability of the phase difference between two signals, evaluated at the same frequency. Signals are said to be coherent at a given frequency if their phase difference is stable. The coherence spectrum displays coherence values for several frequencies. High coherence in a given frequency is often referred to as 'synchrony' or 'phase locking'. The phase lag between the signals need not be zero.

Comodulation map: a 2D heatmap that displays phase-amplitude coupling strength among several frequency components of the LFP signal (see Box 2); also known as 'comodulogram'.

\section{Cross-frequency coupling: an} interaction between specific features of two oscillations of different frequencies, such as instantaneous phase, amplitude, or frequency. There are several possible types of cross-frequency coupling (e.g., phase-phase coupling, phasefrequency coupling, phase-amplitude coupling, amplitude-amplitude coupling).

\section{Local field potential (LFP):}

continuous signal derived from extracellular recordings using intracerebral electrodes. LFPs are generally thought to represent the subthreshold activity of a population of nearby neurons, and would thus reflect synchronous synaptic inputs. The LFP often displays rhythmical activity. Oscillations of different frequency bands usually co-exist in the same LFP signal.

Neuronal oscillations: rhythmic patterns of neuronal activity, which can be detected at different levels through spectral analysis, from intracellular recordings of single neurons to LFP and electroencephalography (EEG) signals. Also known as 'brain waves' or 'brain rhythms'. Oscillations of different frequency bands have been traditionally named using Greek alphabet letters, but other nomenclatures exist (see Box 1). Theta $(\sim 4-12 \mathrm{~Hz})$ and gamma ( 30$160 \mathrm{~Hz}$ ) are two of the most studied neuronal oscillations, though the exact definitions of their frequency ranges remain somewhat arbitrary and vary across laboratories. 
[27] reported synchronization between the hippocampus and $\mathrm{OB}$ at theta frequency during cognitive tasks. However, it remains to be determined whether such synchrony occurs for the classical theta rhythm or respiration-entrained oscillations at theta frequency. At the time, respiration-entrained oscillations were not recognized as an independent activity from theta in the hippocampus.

We note that a similar issue may have happened in studies on delta-frequency oscillations, especially in larger rodent species like rats, which breathe slower than mice. For instance, we suspect that the $4-\mathrm{Hz}$ oscillations described to link the mPFC, the ventral tegmental area, and the hippocampus during an odor-based working memory task in rats [28] may correspond to respiration-entrained oscillations. The origin of $2-5-\mathrm{Hz}$ oscillations in the $\mathrm{mPFC}$ of anesthetized rats has also recently been suggested to be due to respiration [29-32]. Interestingly, a recent work showed oscillations of similar frequency in the mPFC and nucleus accumbens of prairie voles during social interactions [33], whose origin has not been determined. We also believe that the $4-\mathrm{Hz}$ oscillations that emerge in the mPFC and amygdala during freezing behavior in fear-conditioned mice $[34,35]$ are due to respiration. By contrast, sleep-associated slow oscillations have been previously attributed to respiration $[3,36]$, while recent work demonstrated their independence despite the overlapping frequency $[8,14]$.

\section{Theta and Respiration Modulate Distinct Gamma Frequencies}

Research on theta-gamma coupling has been showing that the broad gamma range (30$160 \mathrm{~Hz}$ ) can be divided into sub-bands of independent activity [37-42]. These discoveries were possible due to a modern signal analysis tool, called comodulation map or 'comodulogram' [43] (Box 2). In the hippocampus and parietal cortex of rodents, comodulation maps have shown that theta phase modulates multiple, non-overlapping 'islands' of oscillatory sub-bands within the broad gamma range [37-41]. Moreover, the different gamma sub-bands occur at different theta phases and theta cycles [37-44]. The analysis of phase-amplitude coupling has thus started to unveil a putative functional subdivision of gamma oscillations that has previously not been recognized by power spectral analysis [45]. Furthermore, these studies also showed that cross-frequency coupling patterns depend on the recorded layer along the parietal-CA1-dentate axis $[38,40,41,44]$, as well as on the type of theta-eliciting behavior displayed by the animal (e.g., locomotion vs REM sleep) $[40,41,46]$. Figure 3A (left) shows a representative comodulogram computed for an LFP recorded from the mouse parietal cortex during REM sleep. Notice that the theta phase robustly modulates the amplitude of two nonoverlapping gamma sub-bands: $\sim 40-90-\mathrm{Hz}$ and $\sim 110-160-\mathrm{Hz}$ oscillations [46].

The phase of the respiratory cycle has been long noted to modulate gamma amplitude in the OB [47]. In addition, Fontanini and Bower [4] described bursts of gamma activity entrained by the respiratory cycle in the piriform cortex. More recently, respiration has also been found to modulate local gamma in non-olfactory areas. Namely, in 2014 lto et al. [5] demonstrated that respiration-entrained oscillations (at delta frequency) modulate gamma in the whisker barrel cortex of immobile mice, while respiration-gamma coupling has been reported in the hippocampus of anesthetized mice [6]. In 2017, Biskamp et al. [9] and Zhong et al. [10] independently showed that the mPFC of awake mice exhibits prominent respiration-entrained oscillations and respiration-gamma coupling. Of note, depending on breathing rate, respiration-gamma coupling in the comodulation map can be interpreted as either 'delta'-gamma coupling (during slow breathing) $[9,10]$ or 'theta'-gamma coupling (during fast breathing) [10].

Comodulation maps also reveal that respiration modulates a specific gamma sub-band, which differs from the gamma sub-bands modulated by theta [10] (Figure 3A). Curiously, the
Phase-amplitude coupling: a specific type of cross-frequency coupling in which the instantaneous phase of a slower oscillation modulates the instantaneous amplitude of a faster oscillation. Respiration-entrained oscillations: a pattern of rhythmic LFP activity whose peak frequency matches the frequency of nasal respiration, and which displays high coherence with breathing cycles at the respiration frequency. Also referred to as the 'respiration rhythm' and abbreviated as 'RR'. Though initially described in olfactory areas, $R R$ has been recently detected in several other brain regions. RR disappears when animals breathe through the trachea.

Theta-gamma coupling: usually refers to the phase-amplitude coupling between theta and gamma oscillations, in which the instantaneous amplitude of gamma is modulated by the instantaneous phase of theta. 


\section{Rodents}

(A)
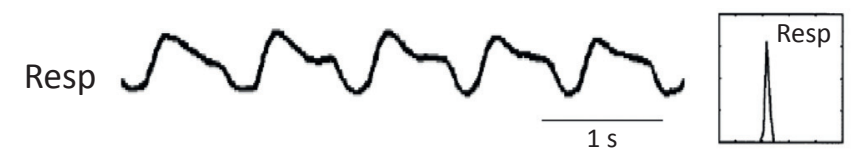

$\mathrm{OB}$

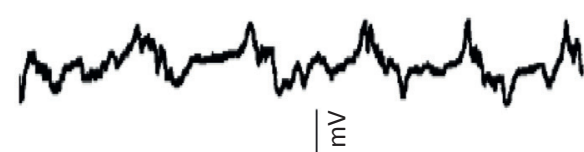

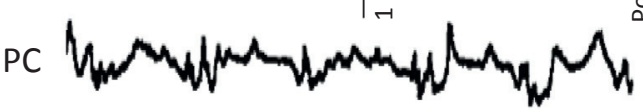

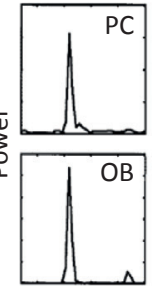

Vm

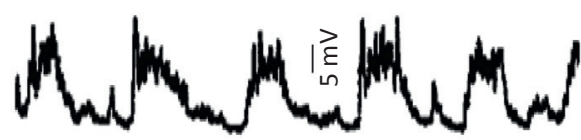

(B)

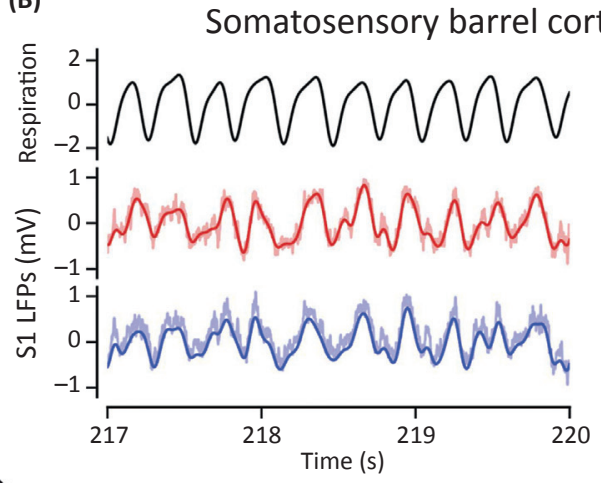

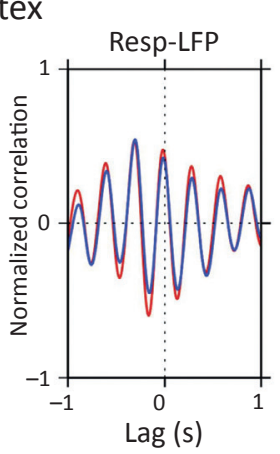

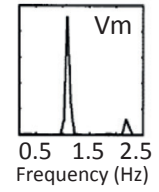

(C) Hippocampus

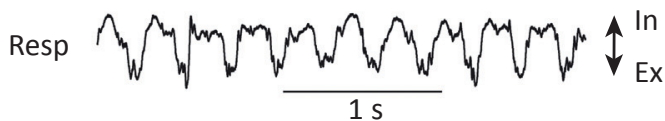

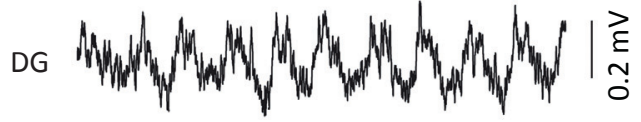

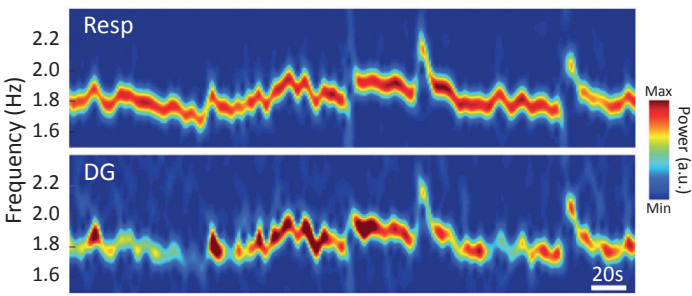

(D)

Prefrontal cortex

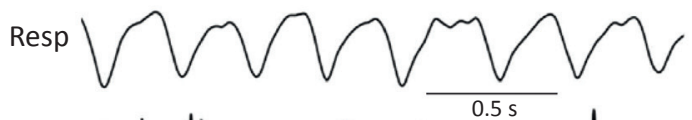

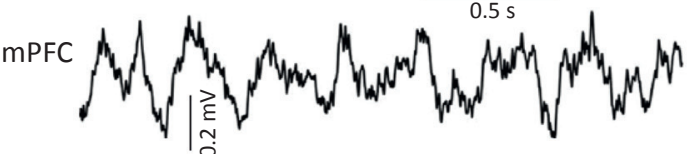
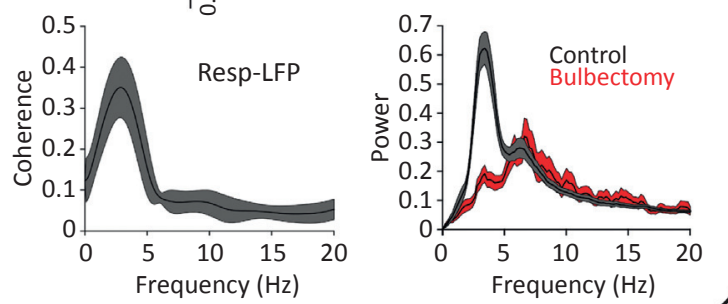

Humans

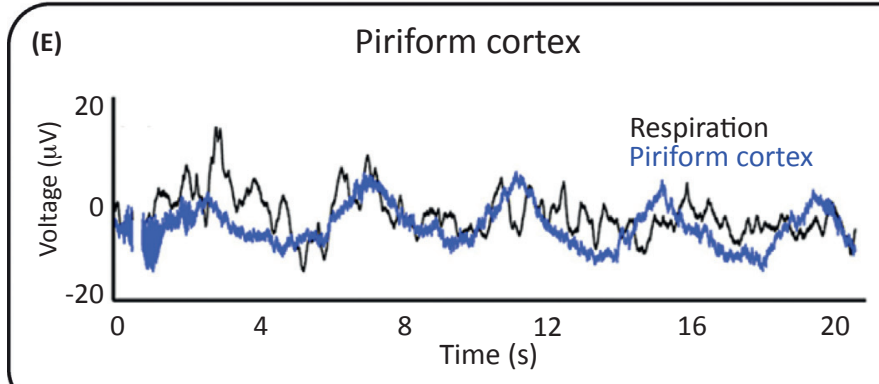

(F)
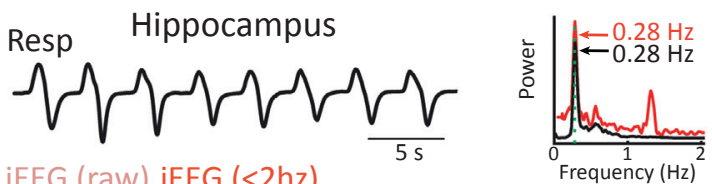

IEEG (raw) iEEG $(<2 \mathrm{hz})$
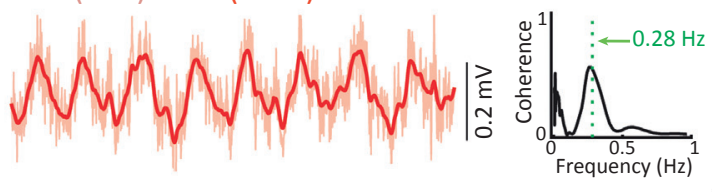

Trends in Neurosciences

Figure 1. Respiration-Entrained LFP Oscillations Are Observed Brain Wide and across Species. (A) Left traces show respiration (Resp) simultaneously recorded with the olfactory bulb (OB) and piriform cortex (PC) LFPs, along with an intracellular recording from a PC neuron (Vm), obtained from a rat anesthetized with ketamine. Right panels show the corresponding power spectral densities. Adapted from [3]. (B) Left traces show respiration (black) and two LFPs (red and blue) simultaneously recorded from the somatosensory whisker barrel cortex of an awake, head-fixed mouse. The right panel shows the cross-correlations between the LFPs and respiration. Adapted from [5]. (C) Top traces show respiration and a simultaneously recorded LFP from the dentate gyrus (DG) of a urethane-anesthetized mouse. Adapted from [6]. The bottom spectrograms show variations in breathing rate and peak frequency of a DG LFP in a rat anesthetized with urethane. Adapted from [8]. (D) Traces show respiration recorded simultaneously with an LFP in the medial prefrontal cortex (mPFC) of an awake immobile mouse. Bottom panels show high LFP- 
frequency range of the respiration-modulated gamma $(\sim 70-120 \mathrm{~Hz})$ lies in between the two gamma sub-bands modulated by theta (compare the left and right panels of Figure 3A). Interestingly, Kay previously reported that the OB has two types of gamma activity - at 35 to $65 \mathrm{~Hz}$ and 65 to $100 \mathrm{~Hz}$ - and that only the faster gamma sub-band is strongly modulated by sniffing cycles [48]. Consistent with Kay's findings, OB spectrograms in Zhong et al. [10] also exhibited a slower type of gamma activity at $40-70 \mathrm{~Hz}$, which was not coupled to respiration and thus not apparent in the comodulogram (see Figure 1 in [10]). In all, these observations suggest that different frequency channels within the broad gamma range could be used for distinct types of information.

\section{Selective Coupling of Gamma Oscillations to Respiration in the Prefrontal Cortex}

The coupling of gamma oscillations to either theta or respiration-entrained LFP rhythms depends on recorded region and behavioral state [10]. For instance, during immobility, the $\mathrm{OB}, \mathrm{mPFC}$, parietal cortex, and hippocampus exhibit coupling between $70-$ and $120-\mathrm{Hz}$ oscillations and respiration [10]. By contrast, during active behaviors such as locomotion, sniffing, and exploration, coupling divides into two regional patterns: the frontal regions ( $\mathrm{OB}$ and mPFC) still exhibit exclusive coupling between respiration and the $70-120-\mathrm{Hz}$ gamma subband, while the hippocampus and the parietal cortex exhibit coupling of the $40-90-\mathrm{Hz}$ and 110-160-Hz gamma sub-bands to theta [10]. Figure 3B (left) shows representative comodulograms computed for each region during exploration, as well as the power spectra for the LFPs and respiration. Note that in the mPFC gamma couples exclusively to respiration but not to theta. This specificity is notable since the LFP in this region contains both these slow oscillations, as visible from the two peaks in the power spectrum. Biskamp et al. [9] reported consistent findings where prominent modulation of $80-100-\mathrm{Hz}$ gamma by respirationentrained oscillations was observed in the mPFC, while theta exhibited no meaningful coupling to gamma in this region.

These recent studies indicate that gamma selectively couples to respiration in the mPFC and warrant revisiting previous reports on theta activity and theta-gamma coupling in frontal regions, which may have detected the respiration-locked rhythm and taken it as theta oscillations. To illustrate this possibility, in Figure 3B (right) we show an example in which respiration occurs at the same frequency as the CA1 theta rhythm. In such cases, the spectral analysis is not able to confidently distinguish between theta, the respiration-entrained rhythm, and superposition of both rhythms. Since respiration is at a similar frequency as the hippocampal theta, one could easily conclude that theta modulates $70-120-\mathrm{Hz}$ oscillations in the mPFC and $\mathrm{OB}$. Because most previous studies have not recorded LFPs concomitantly with respiration, the possibility that their findings were influenced by the respiration-entrained LFP rhythm remains to be determined. The exact frequency range of the modulated gamma activity may give a clue about the nature of the slow oscillation; however, while the precise gamma sub-bands modulated by theta or respiration differ within an animal, there are cases in which they may partially or even substantially overlap, especially when analyzing different animals. Nevertheless, in addition to differences in modulated gamma frequency, there is another physiological feature that may help to separate theta-gamma coupling from respirationgamma coupling, which we discuss next.

respiration coherence (left) and the disappearance of the respiration-entrained rhythm upon bulbectomy (right). Adapted from [9]. (E) Respiration (black) and PC LFP (blue) simultaneously recorded from a patient with intractable epilepsy. Adapted from [11]. (F) Left traces show respiration (black) and intracranial electroencephalogram (iEEG; red lines) from the hippocampus of an epilepsy patient. The right panels depict the power spectra of respiration and hippocampal signals (top), along with their coherence spectrum (bottom). Adapted from [12]. 
(A)

Awake immobility

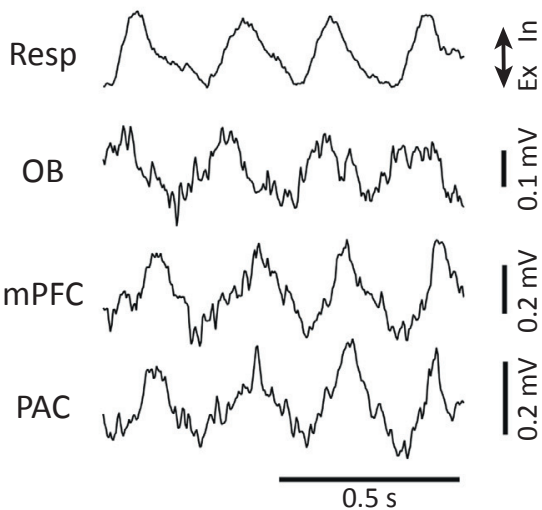

Exploration

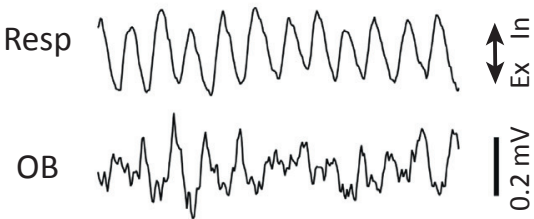

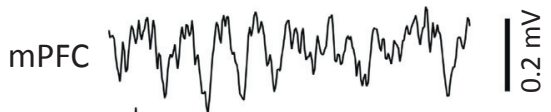

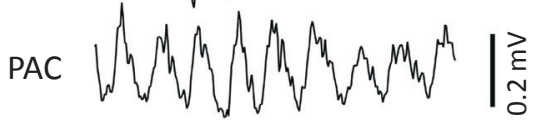

$0.5 \mathrm{~s}$

(B)

REM sleep

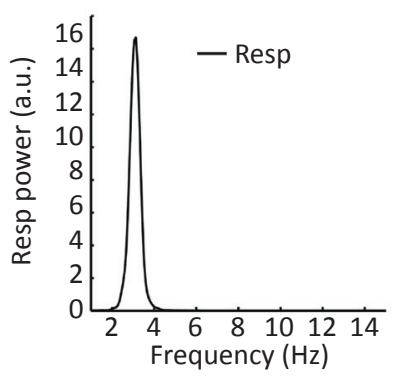

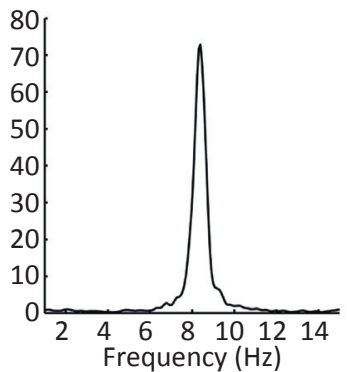

Exploration
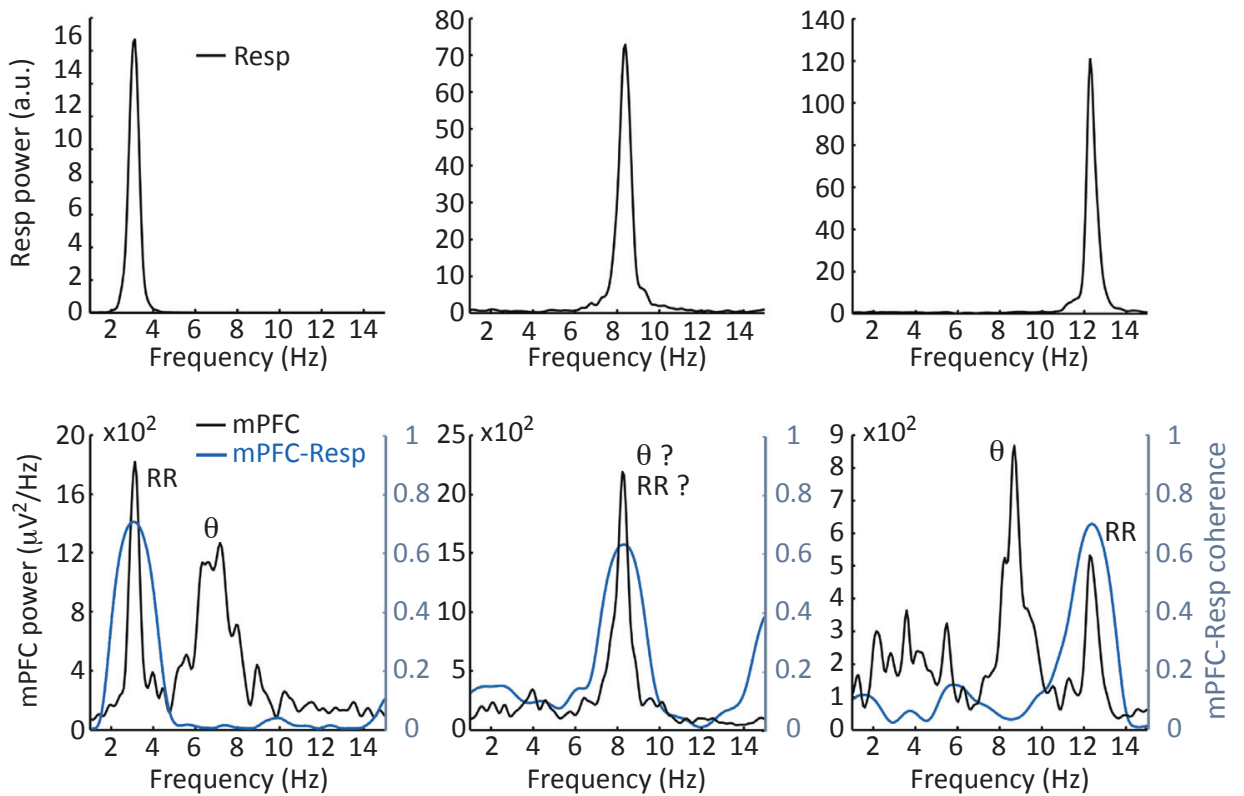

Trends in Neurosciences

Figure 2. Respiration-Entrained LFP Oscillations Co-exist with Theta Oscillations. (A) (Left) Traces show examples of respiration and LFP signals simultaneously recorded during immobility. Notice prominent respirationentrained oscillations in all regions. (Right) As in the left column, but during environment exploration. In this case, respiration-entrained oscillations in $\mathrm{OB}$ are apparent upon visual inspection, but they are less discernible in mPFC and PAC due to the concomitant presence of theta oscillations (notice that theta is slower than respiration in this example). (B) Power spectra (black curves) of respiration (top) and mPFC LFPs (bottom) for three 30-s periods differing in breathing rate $(\sim 3, \sim 8$, and $\sim 12 \mathrm{~Hz}$, respectively, estimated from the peak frequency of the respiration power spectrum). Coherence spectra between the LFP and the respiration signals are also shown (blue curves). The left panels were recorded during REM sleep; the middle and right ones were recorded during environment exploration. In the left and right columns, notice the clear LFP power peaks at the respiration frequency and at theta. Notice further that only the respiration-entrained LFP rhythm is coherent with respiration. In the middle column, respiration occurs at the same frequency as the theta rhythm, and there is only one peak in the LFP power spectrum. Abbreviations: LFP, local field potential; mPFC, medial prefrontal cortex; OB, olfactory bulb; PAC, parietal cortex; REM, rapid eye movement; Resp, respiration; RR, respiration-entrained LFP rhythm; $\theta$, theta rhythm. Data from [10]. 


\section{Box 2. Measuring Phase-Amplitude Coupling}

The strength of phase-amplitude coupling is usually measured between two filtered LFP signals, referred to as a 'frequency pair'. Under this framework, the LFPs are first band-passed in non-overlapping frequency ranges, a 'slow' and a 'fast' oscillation, and the instantaneous phase and amplitude are next extracted (Figure I). A modulation index then estimates the level of coupling between the amplitude of the faster oscillation and the phase of the slower. Though there are many possible metrics, they all tend to rely on searching for consistent variations of the amplitude of the fast oscillation within the cycles of the slow oscillation; for example, a given fast oscillation may have the largest amplitude at the peak of the slow wave. While the modulation index is a single number assessing coupling level of only one frequency pair, another analytical tool - called 'comodulation map' or 'comodulogram' - scans for phase-amplitude coupling across several frequency pairs. It does so by displaying their modulation indices on a pseudocolor 2D map. In this map, the $X$ axis shows the center frequency of the slow oscillation (phase frequency) and the $Y$ axis shows the center frequency of the fast oscillation (amplitude frequency). Warm colors in the comodulation map indicate that the phase of the corresponding frequency in the $X$ axis modulates the amplitude of the corresponding frequency in the $Y$ axis (Figure I).

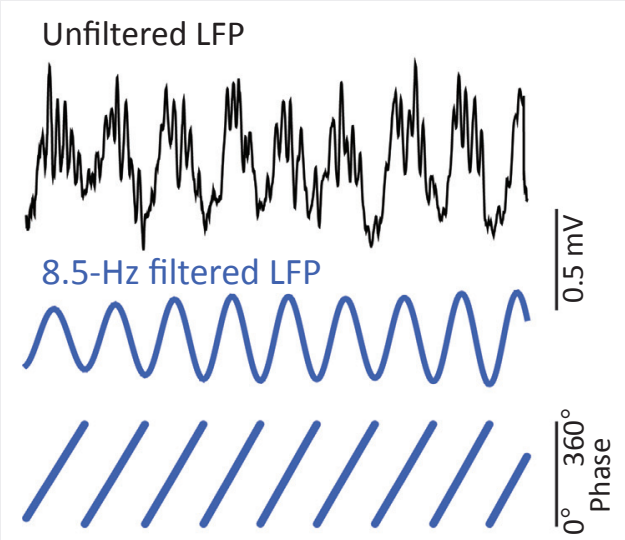

70-Hz filtered LFP
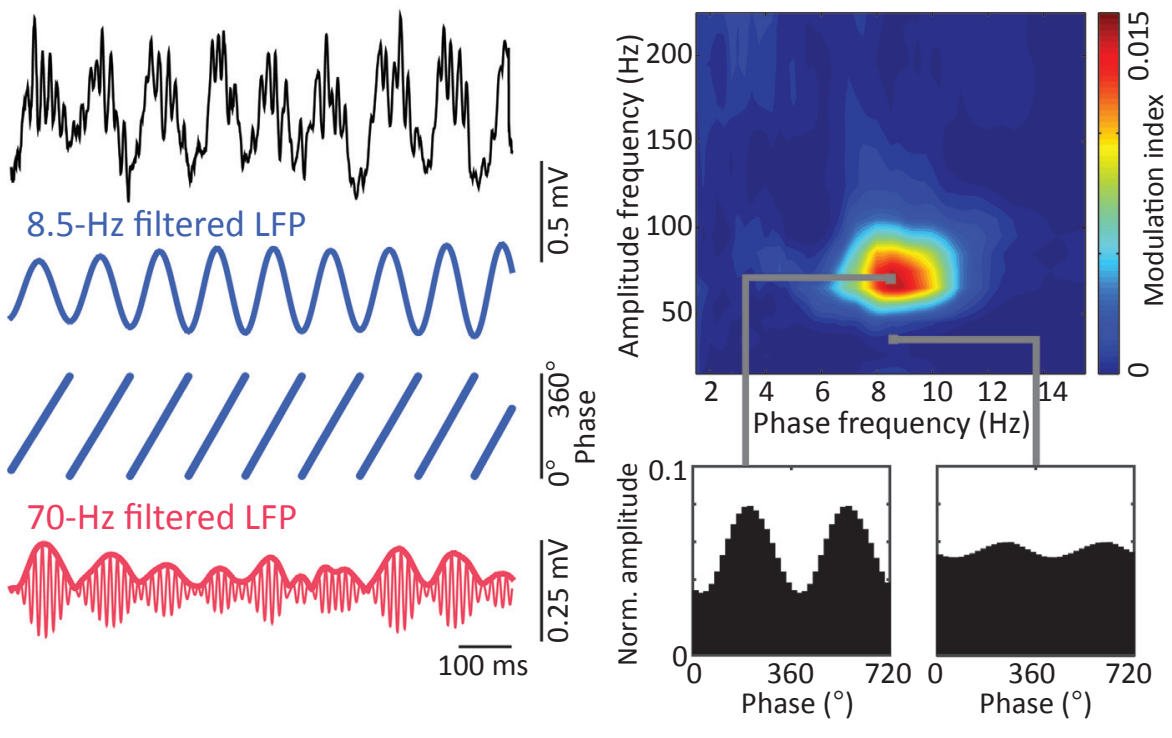

Trends in Neurosciences

Figure I. Assessing Phase-Amplitude Coupling through Comodulation Maps. To compute each entry of the comodulation map (top right), the LFP is band-pass filtered into two ranges: a 'phase frequency' (8-9 Hz in this example) and an 'amplitude frequency' (65-75 Hz in this example; left traces). Next, the phase and amplitude time series of the filtered signals are used to construct a phase-amplitude distribution-like plot; the example bar plots (bottom right) show the distribution of the mean amplitude of the 65-75- $\mathrm{Hz}$ filtered LFP (left) and of the 30-40-Hz filtered LFP (right) over the phases of the $8-9-\mathrm{Hz}$ filtered LFP. A modulation index (MI) then measures the divergence of the phase-amplitude distribution from the uniform distribution (see [43] for details), and is computed for each frequency pair (e.g., $8.5 \mathrm{~Hz}$ and $70 \mathrm{~Hz}$ and $8.5 \mathrm{~Hz}$ and $35 \mathrm{~Hz}$ ). The comodulation map is obtained by expressing Ml values of several frequency pairs by means of a pseudocolor 2D map (top right). This example was obtained from a mouse CA1 recording during active exploration. Adapted, with permission, from [38].

\section{Gamma Coupling to Respiration Decreases during REM Sleep, While Theta- Gamma Coupling Increases}

It has been consistently demonstrated that theta-gamma coupling increases during REM sleep [39-41,46,49,50] (Figure 3C). This effect is most prominent for the theta modulation of the 110$160-\mathrm{Hz}$ gamma sub-band $[40,41,46]$. In stark contrast, respiration-gamma coupling is greatly reduced, or even absent, during REM sleep, despite the fact that the LFP still exhibits the respiration-entrained rhythm [10] (Figure 3C). Interestingly, two recent studies have shown that 'theta'-gamma coupling observed in MPFC during awake states disappears during REM sleep 

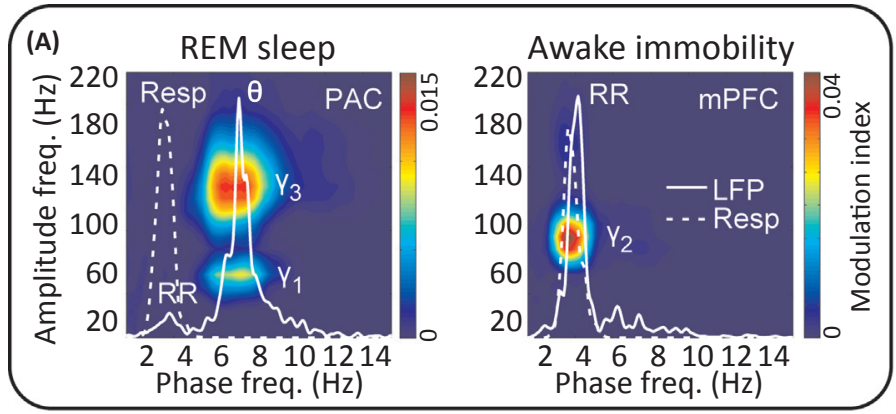

(B)

Exploration
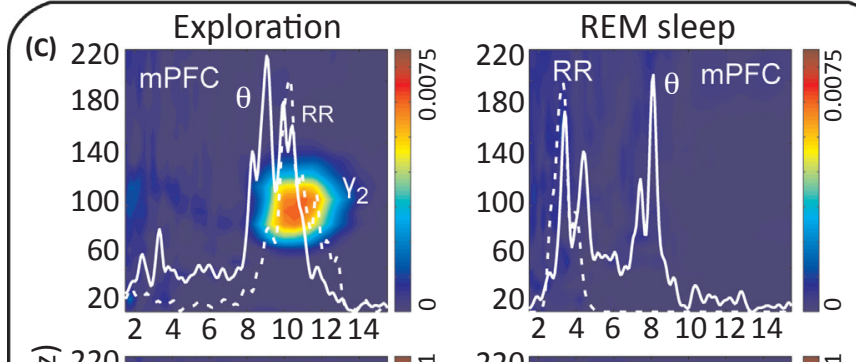

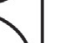

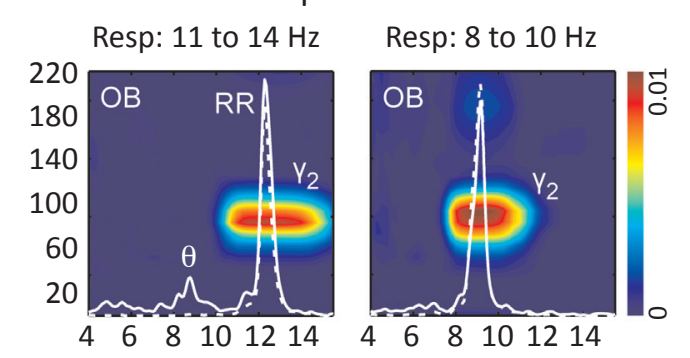

Resp: 8 to $10 \mathrm{~Hz}$
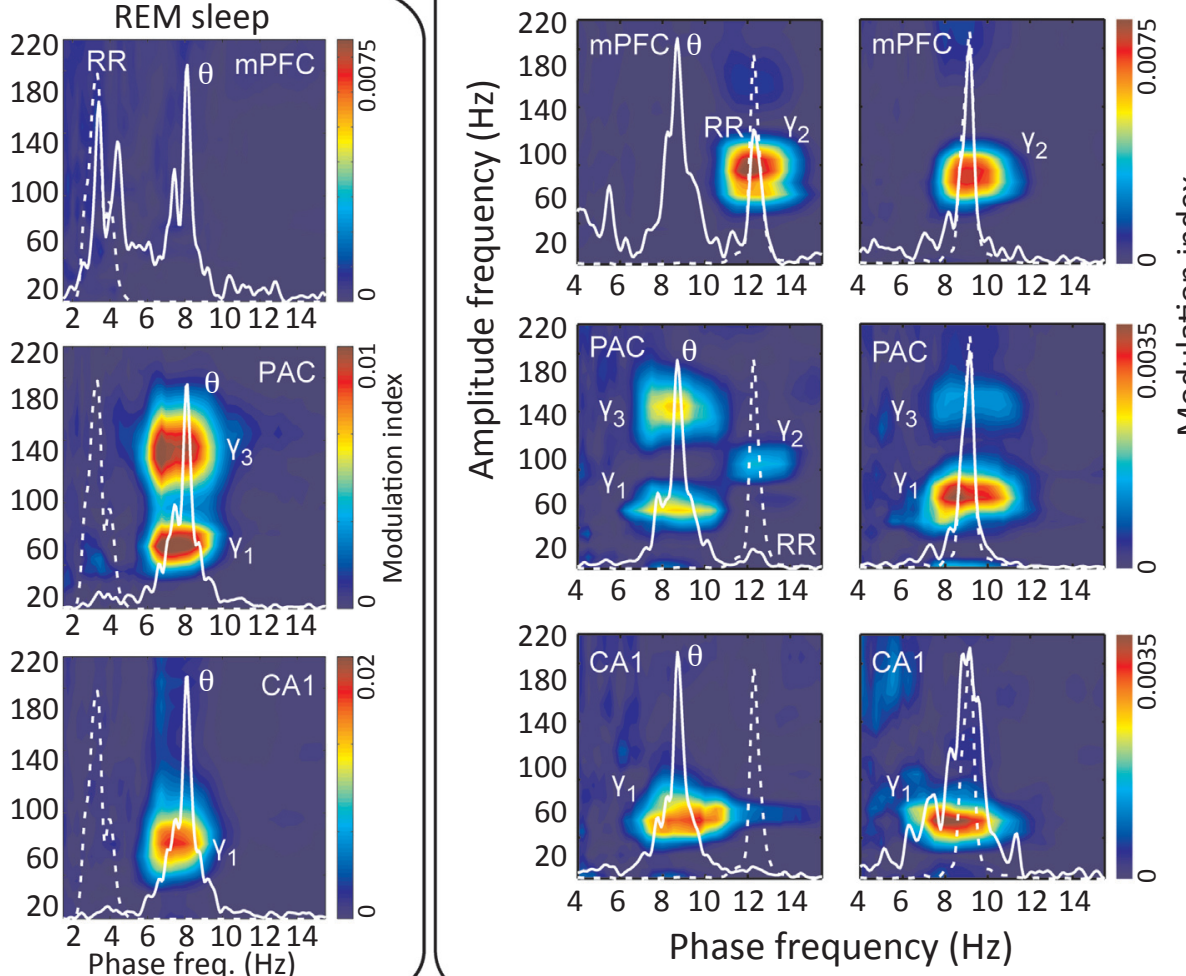

Trends in Neurosciences

Figure 3. Theta and Respiration Modulate Distinct Gamma Frequencies. (A) (Left) Representative comodulogram obtained for a PAC LFP during REM sleep. In this and other plots, the superimposed curves show the power spectrum of the LFP (continuous line) and of the respiration signal (broken line), in arbitrary $Y$ axis units. Theta modulates the amplitude of two gamma sub-bands, $40-90 \mathrm{~Hz}\left(\gamma_{1}\right)$ and $110-160 \mathrm{~Hz}\left(\gamma_{3}\right)$. (Right) As in the left panel, but for an mPFC LFP recorded during immobility. RR modulates the amplitude of $70-120-\mathrm{Hz}$ oscillations $\left(\gamma_{2}\right)$. (B) Comodulograms computed for multisite LFP recordings. The left column was obtained for an epoch in which respiration was faster than theta. Notice that theta couples to $\gamma_{1}$ and $\gamma_{3}$, while RR couples to $\gamma_{2}$. The right column was obtained for an epoch in which respiration occurred at the same frequency as theta. In this case, RR- $\gamma_{2}$ in mPFC is likely to be mistaken as theta- $\gamma_{2}$ coupling if respiration is not simultaneously recorded. (C) Comodulograms computed for multisite LFP recordings during exploration (left) and REM sleep (right). During REM sleep, $\theta-\gamma_{1}$ and $\theta-\gamma_{3}$ coupling increase in PAC and CA1, while RR- $\gamma_{2}$ coupling disappears in mPFC. Data from [10]. Abbreviations: LFP, local field potential; mPFC, medial prefrontal cortex; PAC, parietal cortex; REM, rapid eye movement; Resp, respiration; RR, respiration-entrained LFP rhythm; $\theta$, theta.

$[19,23]$. Though respiration was not concomitantly recorded in these studies, given the negative dependence on REM sleep, we suspect that the coupling pattern observed during awake states actually corresponded to respiration-gamma coupling, not theta-gamma coupling. Thus, when respiration is not directly monitored, two features may provide hints as to the nature of the slow oscillation: the precise frequency band of the modulated gamma oscillation 
and the dependence of coupling on REM sleep. We note, however, that such differences have only been reported for mice and have yet to be shown for rats and other rodents.

\section{Potential Mechanisms and Functions of Respiration-Entrained Rhythms}

Neuronal networks in olfactory regions such as the $\mathrm{OB}$ and piriform cortex exhibit strong synchronization with breathing cycles, as inferred by both LFP and spiking activity $[1-4,17,36]$. Previous work has shown that such phase locking depends on re-afferent inputs originating in sensory epithelia in the nasal cavity, as opposed to central pattern generators in brain-stem circuits responsible for efferent motor control of breathing [3]. Of note, while neuronal activity locked to sensory afferents depends on nasal airflow, the stimulating air need not contain odorants [51]. Current evidence indicates that respiration-entrained oscillations in non-olfactory regions also depend on sensory signals from the nasal cavity - and not on respiratory central pattern generators. For instance, tracheotomy or inactivation of the OB hinders respirationlocked activity in the somatosensory cortex [5], hippocampus [6,8], and mPFC [9] (see Figure 1D), while the animals still display the motor act of breathing. Furthermore, neuronal activity in both olfactory and non-olfactory areas can be entrained by rhythmical delivery of air puffs into the nasal cavity $[3,8,52]$. The primary olfactory areas are the natural candidates for relaying the respiratory rhythmicity to further downstream regions. In some cases, well-known anatomical connections exist, such as direct connections from the piriform cortex to the prefrontal cortex [53], and indirect connections to the hippocampus through the entorhinal cortex [54]. Other areas, however, such as the whisker barrel cortex, are likely to depend on multisynaptic pathways [5].

The mechanisms underlying respiration-gamma coupling are presently unknown. To gain insight into this question, Heck et al. [55] proposed a graph model of excitatory and inhibitory cells coupled via abundant short and sparse long-range connections, to recapitulate the architecture of cortical networks. The effect of respiration was simulated as a sinusoidal input to the model; the respiratory input caused perturbations that could bring trajectories into a region of the phase space containing a gamma-period limit cycle (during inspiration) or out of it (during expiration) to a fixed point. This model thus provides a proof of principle that modulation of gamma amplitude by the breathing cycle can potentially be achieved by intrinsic properties of cortical networks, assuming that respiration drives neuronal excitability. Although respiration does modulate spike probability in several brain regions [5-7,9], the latter was phenomenologically modeled in Heck et al. [55], and it remains to be determined what would be its biophysical/synaptic origins. An alternative hypothesis is that gamma would be generated by phasic GABAergic transmission from inhibitory interneurons and that these cells would be directly modulated by respiration-locked inputs from olfactory areas. Interneurons have been classically linked to gamma [56-58], but less is known about how exactly the different gamma sub-bands would relate to the diversity of interneuron subtypes $[59,60]$. Moreover, the existence of olfactory-related inputs impinging on interneurons in widespread regions of the brain has yet to be demonstrated.

It is interesting to note that in humans, some breathing practices, such as those performed by meditators, have been shown to influence emotional states and cognitive function [61-63]. Irrespective of the underlying mechanisms, it is possible that nasal respiration affects brain functioning by driving slow oscillations and modulating the amplitude of fast ones [5-10]. Consistent with this possibility, current evidence suggests that brain oscillations and their cross-frequency coupling play functional roles [64-67]. Slow oscillations - as the respirationentrained rhythm - tend to be coherent over large distances and would be well suited for aiding interregional communication [67], while faster, gamma-frequency oscillations have been 
associated with local computations [45,68]. Respiration-gamma coupling could thus serve as a means of integrating locally generated gamma assemblies across distant regions, akin to what has been previously proposed for theta-gamma coupling [66,67]. Importantly, since theta and respiration modulate distinct gamma frequencies, different types of information could be simultaneously transmitted across the brain without interference [10]. While this is all hypothetical, the bottom line is that understanding the effects of respiration on the brain may intrinsically relate to understanding what brain oscillations do.

Although most findings so far on respiration-entrained rhythms have been obtained from mice and rats, two recent studies have shown electrical activity locked to breathing cycles in the human brain [11,12], which, similar to rodents, depends on nasal airflow [11]. Interestingly, these studies also showed that the breathing cycle modulates the amplitude of higherfrequency oscillations in intracranial electroencephalographies (EEGs) [11,12], and that breathing route (nasal vs oral) influences cognitive performance in healthy subjects [11]. Of note, cross-frequency coupling of the phase-amplitude type has been well established in humans and previously linked to sensory, motor, and cognitive functions [66,67,69-72]. Therefore, the fact that the amplitude of faster oscillations couples to the respiratory cycle $[11,12]$ is consistent with the idea that nasal breathing may modulate higher brain functions [55]

\section{Concluding Remarks}

Here we revisited recent findings on respiration-entrained LFP rhythms and respiration-gamma coupling. We draw the following conclusions:

- Respiration-entrained LFP oscillations occur in several brain regions, and are particularly prominent in the frontal lobe.

- Depending on breathing rate, respiration-entrained LFP oscillations may have identical frequency like theta oscillations, and therefore be mistaken as such; at low breathing rate, they may also be mistaken as delta or '4-Hz oscillations'.

- The frequencies of the gamma sub-bands modulated by theta and respiration differ; in the prefrontal cortex, gamma oscillations at $\sim 70-120 \mathrm{~Hz}$ couple to respiration, not theta.

- Respiration-gamma coupling can be seen either during immobility in the absence of theta and at low respiration rates or during exploration in the presence of theta and at high respiration rates.

- During REM sleep, respiration-gamma coupling disappears while theta-gamma coupling increases.

In summary, recent evidence shows that respiration-locked LFP oscillations are ubiquitous in the brain. We advocate for awareness of these entrainment effects when studying neural circuitry, so that the respiration-locked oscillations are not mistaken for another rhythm, such as delta or theta. To confidently identify respiration-entrained rhythms, we recommend tracking nasal respiration and assessing its coherence with LFP activity. In the absence of respiration recordings, the modulated gamma frequency and coupling dependence on REM sleep may give clues about the nature of the slow rhythm.

Respiration-coupled oscillations, given their recently recognized presence in widespread regions of the rodent brain and capacity of modulating gamma activity [5-10], are likely to constitute a means for linking distributed cell assemblies, akin to functions commonly attributed to the theta rhythm. This hypothesis should be tested in the years to come (see Outstanding Questions). At any event, being able to identify this often overlooked slow rhythm is a first crucial step toward better understanding its functional role.

\section{Outstanding Questions}

What are the functions of LFP oscillations entrained by nasal respiration? Do they play cognitive roles? Do they have the same function(s) in the different brain regions where they have been detected? Similar questions apply for respiration-gamma coupling.

Are the '4-Hz oscillations' reported in the $\mathrm{mPFC}$ and amygdala during fear learning respiration-entrained oscillations? What about the $4-\mathrm{Hz}$ activity described to link the hippocampus, ventral tegmental area, and mPFC during working memory?

Respiration-entrained LFP oscillations have been described in several regions of the rodent brain while animals breathed room air. However, given their nature, one could wonder if they have any special relation to olfaction and odor-related tasks. Would this be the case?

Within the same brain region, respiration-entrained oscillations are not always present, but rather occur intermittently even for the same behavioral state. What explains the intermittency of respiration-entrained oscillations? What are the main triggers leading to their appearance?

What are the biophysical mechanisms underlying LFP oscillations locked to nasal respiration? What synapses, transmitters, and patterns of network connectivity are involved? Similarly, what are the network mechanisms underlying respiration-gamma coupling?

Why respiration-gamma coupling substantially decreases during REM sleep? What are the underlying cellular- and network-level mechanisms?

Would the different gamma frequencies modulated by theta and respiration translate to different coding contents? How are these different gamma frequencies generated?

As delta and theta, the respirationentrained oscillations seem to be a global brain rhythm. How many global rhythms does the brain produce? 


\section{Acknowledgments}

This work was supported by the Deutsche Forschungsgemeinschaft (SFB 636/B06; SFB 1134/A01; Dr 326/10-1), Bundesministerium für Bildung und Forschung (Bernstein Center for Computational Neurosciences, No. 01GQ1003A German-Brazil Cooperation grant: No. 01DN12098), the Brazilian National Council for Scientific and Technological Development (CNPq), the Brazilian Coordination for the Improvement of Higher Education Personal (CAPES), and the Alexander von Humboldt Foundation. Figures 2 and 3 were generated de novo using data from [10].

\section{References}

1. Adrian, E.D. (1942) Olfactory reactions in the brain of the hedgehog. J. Physiol. 100, 459-473

2. Kay, L.M. et al. (2009) Olfactory oscillations: the what, how and what for. Trends Neurosci. 32, 207-214

3. Fontanini, A. et al. (2003) Ketamine-xylazine-induced slow $(<1.5 \mathrm{~Hz})$ oscillations in the rat piriform (olfactory) cortex are functionally correlated with respiration. J. Neurosci. 23, 7993-8001

4. Fontanini, A. and Bower, J.M. (2005) Variable coupling between olfactory system activity and respiration in ketamine/xylazine anesthetized rats. J. Neurophysiol. 93, 3573-3581

5. Ito, J. et al. (2014) Whisker barrel cortex delta oscillations and gamma power in the awake mouse are linked to respiration. Nat. Commun. 5, 3572

6. Yanovsky, Y. etal. (2014) Slow oscillations in the mouse hippocampus entrained by nasal respiration. J. Neurosci. 34, 5949-5964

7. Nguyen Chi, V. et al. (2016) Hippocampal respiration-driven rhythm distinct from theta oscillations in awake mice. J. Neurosci. 36, 162-17

8. Lockmann, A.L.V. et al. (2016) A respiration-coupled rhythm in the rat hippocampus independent of theta and slow oscillations. J. Neurosci. 36, 5338-5352

9. Biskamp, J. et al. (2017) Organization of prefrontal network activity by respiration-related oscillations. Sci. Rep. 7, 45508

10. Zhong, W. et al. (2017) Selective entrainment of gamma subbands by different slow network oscillations. Proc. Natl. Acad. Sci. U. S. A. 114, 4519-4524

11. Zelano, C. et al. (2016) Nasal respiration entrains human limbic oscillations and modulates cognitive function. J. Neurosci. 36, 12448-12467

12. Herrero, J.L. et al. (2018) Breathing above the brainstem: volitional control and attentional modulation in humans. J. Neurophysiol. 119, 145-159

13. Liu, Y. et al. (2017) Hippocampal sharp-wave ripples in awake mice are entrained by respiration. Sci. Rep. 7, 8950

14. Viczko, J. et al. (2014) Lack of respiratory coupling with neocortical and hippocampal slow oscillations. J. Neurosci. 34, 3937-3946

15. Lockmann, A.L.V. and Belchior, H. (2014) New insights into the role of respiratory inputs in hippocampal oscillations. J. Neurosci. 34, 9473-9475

16. Wesson, D.W. et al. (2008) Sniffing behavior of mice during performance in odor-guided tasks. Chem. Senses 33, 581-596

17. Rojas-Líbano, D. et al. (2014) The olfactory bulb theta rhythm follows all frequencies of diaphragmatic respiration in the freely behaving rat. Front. Behav. Neurosci. 8, 214

18. Stujenske, J.M. et al. (2014) Fear and safety engage competing patterns of theta-gamma coupling in the basolateral amygdala. Neuron 83, 919-933

19. Zhang, X. et al. (2016) Impaired theta-gamma coupling in APPdeficient mice. Sci. Rep. 6, 21948

20. Adhikari, A. et al. (2010) Synchronized activity between the ventral hippocampus and the medial prefrontal cortex during anxiety. Neuron 65, 257-269

21. Lesting, J. et al. (2011) Patterns of coupled theta activity in amygdala-hippocampal-prefrontal cortical circuits during fear extinction. PLoS One 6, e21714

22. Zheng, C. and Zhang, T. (2015) Synaptic plasticity-related neural oscillations on hippocampus-prefrontal cortex pathway in depression. Neuroscience 292, 170-180
23. Pavlovsky, V.A. et al. (2017) Dopamine modulates delta-gamma phase-amplitude coupling in the prefrontal cortex of behaving rats. Front. Neural Circuits 11, 29

24. Seidenbecher, T. et al. (2003) Amygdalar and hippocampal theta rhythm synchronization during fear memory retrieval. Science $301,846-850$

25. Buzsáki, G. (2002) Theta oscillations in the hippocampus. Neuron 33, 325-340

26. Macrides, F. et al. (1982) Temporal relationship between sniffing and the limbic theta rhythm during odor discrimination reversa learning. J. Neurosci. 2, 1705-1717

27. Kay, L.M. (2005) Theta oscillations and sensorimotor perfor mance. Proc. Natl. Acad. Sci. U. S. A. 102, 3863-3868

28. Fujisawa, S. and Buzsáki, G. (2011) A 4 Hz oscillation adaptively synchronizes prefrontal, VTA, and hippocampal activities. Neuron 72, 153-165

29. Roy, A.etal. (2017)Prefrontal-hippocampal coupling bythetarhythm and by $2-5 \mathrm{~Hz}$ oscillation in the delta band: the role of the nucleus reuniens of the thalamus. Brain Struct. Funct. 222, 2819-2830

30. Bagur, S. and Benchenane, K. (2018) Taming the oscillatory zoo in the hippocampus and neo-cortex: a review of the commentan of Lockmann and Tort on Roy et al. Brain Struct. Funct. 223, 5-9

31. Lockmann, A.L.V. and Tort, A.B.L. (2018) Nasal respiration entrains delta-frequency oscillations in the prefrontal cortex and hippocampus of rodents. Brain Struct. Funct. 223, 1-3

32. Kocsis, B. et al. (2018) Respiration-coupled rhythms in prefronta cortex: beyond if, to when, how, and why. Brain Struct. Funct. 223, 11-16

33. Amadei, E.A. et al. (2017) Dynamic corticostriatal activity biases social bonding in monogamous female prairie voles. Nature 546 297-301

34. Dejean, C. et al. (2016) Prefrontal neuronal assemblies temporally control fear behaviour. Nature 535, 420-424

35. Karalis, N. et al. (2016) 4-Hz oscillations synchronize prefrontalamygdala circuits during fear behavior. Nat. Neurosci. 19, 605-612

36. Fontanini, A. and Bower, J.M. (2006) Slow-waves in the olfactory system: an olfactory perspective on cortical rhythms. Trends Neurosci. 29, 429-437

37. Tort, A.B.L. et al. (2008) Dynamic cross-frequency couplings of local field potential oscillations in rat striatum and hippocampus during performance of a T-maze task. Proc. Natl. Acad. Scl. U. S. A. 105, 20517-20522

38. Scheffer-Teixeira, R. et al. (2012) Theta phase modulates multiple layer-specific oscillations in the CA1 region. Cereb. Cortex 22 2404-2414

39. Schomburg, E.W. et al. (2014) Theta phase segregation of inputspecific gamma patterns in entorhinal-hippocampal networks. Neuron 84, 470-485

40. Scheffer-Teixeira, R. and Tort, A.B.L. (2017) Unveiling fast field oscillations through comodulation. eNeuro 4, ENEURO.0079-17.2017

41. Tort, A.B.L. et al. (2013) Theta-associated high-frequency oscil lations $(110-160 \mathrm{~Hz})$ in the hippocampus and neocortex. Prog. Neurobiol. 100, 1-14

42. Colgin, L.L. et al. (2009) Frequency of gamma oscillations routes flow of information in the hippocampus. Nature 462, 353-357

43. Tort, A.B.L. et al. (2010) Measuring phase-amplitude coupling between neuronal oscillations of different frequencies. J. Neurophysiol. 104, 1195-1210
What are the functional differences among them?

To what degree are respirationentrained LFP oscillations evolutionary conserved across mammals? Do nonmammalian species also have them? 
44. Lasztóczi, B. and Klausberger, T. (2014) Layer-specific GABAergic control of distinct gamma oscillations in the CA1 hippocampus. Neuron 81, 1126-1139

45. Buzsáki, G. and Wang, X.-J. (2012) Mechanisms of gamma oscillations. Annu. Rev. Neurosci. 35, 203-225

46. Scheffzük, C. et al. (2011) Selective coupling between theta phase and neocortical fast gamma oscillations during REM-sleep in mice. PLoS One 6, e28489

47. Rojas-Líbano, D. and Kay, L.M. (2008) Olfactory system gamma oscillations: the physiological dissection of a cognitive neural system. Cogn. Neurodyn. 2, 179-194

48. Kay, L.M. (2003) Two species of gamma oscillations in the olfactory bulb: dependence on behavioral state and synaptic interactions. J. Integr. Neurosci. 2, 31-44

49. Scheffzük, C. et al. (2013) Global slowing of network oscillations in mouse neocortex by diazepam. Neuropharmacology 65 , 123-133

50. Brankačk, J. et al. (2012) Distinct features of fast oscillations in phasic and tonic rapid eye movement sleep. J. Sleep Res. 21, 630-633

51. Grosmaitre, X. et al. (2007) Dual functions of mammalian olfactory sensory neurons as odor detectors and mechanical sensors. Nat. Neurosci. 10, 348-354

52. Wu, R. et al. (2017) Activity patterns elicited by aiflow in the olfactory bulb and their possible functions. J. Neurosci. 37, 10700-10711

53. Clugnet, M.-C. and Price, J.L. (1987) Olfactory input to the prefrontal cortex in the rat. Ann. N. Y. Acad. Sci. 510, 231-235

54. Wilson, R.C. and Steward, O. (1978) Polysynaptic activation of the dentate gyrus of the hippocampal formation: an olfactory input via the lateral entorhinal cortex. Exp. Brain Res. 33, 523-534

55. Heck, D.H. et al. (2017) Breathing as a fundamental rhythm of brain function. Front. Neural Circuits 10, 115

56. Bartos, M. et al. (2002) Fast synaptic inhibition promotes synchronized gamma oscillations in hippocampal interneuron networks. Proc. Natl. Acad. Sci. U. S. A. 99, 13222-13227

57. Bartos, M. et al. (2007) Synaptic mechanisms of synchronized gamma oscillations in inhibitory interneuron networks. Nat. Rev. Neurosci. 8, 45

58. Wang, X.-J. and Buzsáki, G. (1996) Gamma oscillation by synaptic inhibition in a hippocampal interneuronal network model. J. Neurosci. 16, 6402-6413
59. Whittington, M.A. and Traub, R.D. (2003) Interneuron diversity series: inhibitory interneurons and network oscillations in vitro. Trends Neurosci. 26, 676-682

60. Klausberger, T. and Somogyi, P. (2008) Neuronal diversity and temporal dynamics: the unity of hippocampal circuit operations. Science 321, 53-57

61. Lutz, A. et al. (2004) Long-term meditators self-induce highamplitude gamma synchrony during mental practice. Proc. Natl. Acad. Sci. U. S. A. 101, 16369-16373

62. Brown, R.P. and Gerbarg, P.L. (2005) Sudarshan Kriya yogic breathing in the treatment of stress, anxiety, and depression: part I-neurophysiologic model. J. Altern. Complement. Med. 11, 189-201

63. Hölzel, B.K. et al. (2007) Differential engagement of anterior cingulate and adjacent medial frontal cortex in adept meditators and non-meditators. Neurosci. Lett. 421, 16-21

64. Buzsaki, G. (2006) Rhythms of the Brain, Oxford University Press

65. Wang, X.-J. (2010) Neurophysiological and computational principles of cortical rhythms in cognition. Physiol. Rev. 90, $1195-1268$

66. Canolty, R.T. and Knight, R.T. (2010) The functional role of crossfrequency coupling. Trends Cogn. Sci. 14, 506-515

67. Hyafil, A. et al. (2015) Neural cross-frequency coupling: connecting architectures, mechanisms, and functions. Trends Neurosci. $38,725-740$

68. Sirota, A. et al. (2008) Entrainment of neocortical neurons and gamma oscillations by the hippocampal theta rhythm. Neuron 60 , 683-697

69. Canolty, R.T. et al. (2006) High gamma power is phase-locked to theta oscillations in human neocortex. Science 313, 1626-1628

70. Cohen, M.X. et al. (2009) Oscillatory activity and phase-amplitude coupling in the human medial frontal cortex during decision making. J. Cogn. Neurosci. 21, 390-402

71. Axmacher, N. et al. (2010) Cross-frequency coupling supports multi-item working memory in the human hippocampus. Proc. Natl. Acad. Sci. U. S. A. 107, 3228-3233

72. Staresina, B.P. et al. (2015) Hierarchical nesting of slow oscilla tions, spindles and ripples in the human hippocampus during sleep. Nat. Neurosci. 18, 1679

73. Brankačk, J. et al. (1993) Current source density analysis of the hippocampal theta rhythm: associated sustained potentials and candidate synaptic generators. Brain Res. 615, 310-327 